\title{
SUL PRINCIPIO DI HUYGHENS
}

“Nuovo Cimento » ser. $3^{\text {a }}$, vol. XXXI, I 892, pp. 244-255; vol. XXXII, 1892, pp. 59-65; vol. XXXIII, I893, pp. 32-36 e pp. 7 I-77.

Avendo la Direzione di questo giornale desiderato un resoconto delle pubblicazioní di Kirchhoff, Beltrami, Poincarke, uscite recentemente, nelle quali si tratta del principio di HUYGHENS, pubblico la seguente lezione di un corso fatto in quest'anno sulla elasticità e l'ottica, la quale riassume i detti lavori. Per norma dei lettori, osservo che la presente lezione faceva parte del capitolo sulle vibrazioni dei fluidi elastici, seguiva la teoria delle onde piane e sferiche, ed era premessa al capitolo in cui doveva esporsi la teoria di HELMHOLTZ, sui tubi sonori. L'applicazione all'ottica dei risultati ottenuti derivava immediatamente dalla dimostrazione che anche le vibrazioni trasversali nei mezzi isotropi dipendono dalla equazione differenziale (I).

Prof. Vito VolterRa.

\section{L'equazione differenziale}

$$
\frac{\partial^{2} \theta}{\partial t^{2}}=a^{2} \Delta^{2} \theta
$$

a cui soddisfano il potenziale di velocità ed il potenziale degli spostamenti di un fluido elastico vibrante, ammette l'integrale trovato da EULERO

$$
\theta=\frac{f(r+a t)+\varphi(r-a t)}{r}
$$

essendo $r=\sqrt{\left(x-x_{0}\right)^{2}+\left(y-y_{0}\right)^{2}+\left(z-z_{0}\right)^{2}}$ e denotando con $f$ e $\varphi$ due funzioni arbitrarie. Questo integrale corrisponde ad onde sferiche progressive e regressive il cui centro è il punto $x_{\circ} y_{\mathrm{o}} z_{\mathrm{o}}$, le quali si propagano colla velocità $a$.

Cambiando il centro e la funzione arbitraria $\varphi$, otterremo infiniti integrali particolari della (I), e siccome questa equazione è lineare, così potremo avere un nuovo integrale sommando un numero qualunque di queste soluzioni particolari. Si avrà dunque come integrale della (I)

$$
\theta=\sum_{i}^{n} \frac{\varphi_{2}\left(r_{i}-a t\right)}{r_{i}}
$$

essendo $r_{i}=\sqrt{\left(x-x_{i}\right)^{2}+\left(y-y_{i}\right)^{2}+\left(z-z_{i}\right)^{2}}$ e rappresentando con $\varphi_{i}$ delle funzioni arbitrarie. Se queste funzioni saranno regolari, $\theta$ e le sue derivate non avranno delle singolarità che nei centri $x_{i} y_{i} z_{i}$.

Consideriamo il caso di due soli centri A, B. Siano $x_{\mathrm{r}} y_{\mathrm{r}} z_{\mathrm{I}}$ le coordinate di $\mathrm{A}$, e si denoti con $s_{1}$ il segmento $\mathrm{AB}, \operatorname{con} \alpha, \beta, \gamma$ i suoi coseni di direzione. 
Le coordinate di B saranno allora

quindi

$$
x_{2}=x_{I}+\alpha s_{I} \quad, \quad y_{2}=y_{I}+\beta s_{I} \quad, \quad z_{2}=z_{I}+\gamma s_{I}
$$

Si prenda

$$
\theta=\frac{\varphi_{1}\left(r_{1}-a t\right)}{r_{1}}+\frac{\varphi_{2}\left(r_{2}-a t\right)}{r_{2}} .
$$

$$
-\varphi_{I}=\varphi_{2}=\frac{\varphi}{s_{I}}
$$

Avremo

$$
\theta=\frac{I}{s_{I}}\left[\frac{\varphi\left(r_{2}-a t\right)}{r_{2}}-\frac{\varphi\left(r_{1}-a t\right)}{r_{I}}\right] .
$$

Facciamo ora avvicinare indefinitamente il punto $\mathrm{B}$ al punto $\mathrm{A}$ diminuendo $s_{I}$ e conservando costanti $\alpha, \beta, \gamma$. Otterremo

$$
\begin{aligned}
& \theta_{I}=\lim _{s_{I}=0} \theta=\lim _{s_{I}=0} \frac{I}{s_{I}}\left[\frac{\varphi\left(r_{2}-a t\right)}{r_{I}}-\frac{\varphi\left(r_{I}-a t\right)}{r_{I}}\right]=\frac{d}{d s_{I}} \frac{\varphi\left(r_{I}-a t\right)}{r_{I}} \\
& =\left[\frac{\varphi^{\prime}\left(r_{I}-a t\right)}{r_{I}}-\frac{\varphi\left(r_{I}-a t\right)}{r_{I}^{2}}\right]\left[\frac{\left(x_{I}-x\right) \alpha+\left(y_{I}-y\right) \beta+\left(z_{I}-z\right) \gamma}{r_{I}}\right] .
\end{aligned}
$$

Si trova in tal modo un nuovo integrale della equazione (I). Se esaminiamo il moto corrispondente al potenziale di velocità $\theta_{x}$, si vede facilmente che esso è un movimento che si propaga per onde sferiche il cui centro è il punto $x_{x} y_{x} z_{x}$. Nello stesso modo calcolando

$$
\theta_{2}=\frac{d \theta_{1}}{d s_{2}}
$$

essendo $s_{2}$ una direzione eguale o diversa da $s_{1}$, otterremo un nuovo integrale della equazione il quale corrisponderà ad un movimento che esso pure si propaga dallo stesso centro per onde sferiche. Si vede dunque che può ottenersi una generalizzazione dei moti che si propagano per onde sferiche prendendo per potenziale di velocità

$$
\theta_{p}=\frac{d p}{d s_{x} d s_{2} \cdots d s_{p}} \theta
$$

essendo $\theta=\varphi\left(r_{I}-a t\right) / r_{I}$, ed $s_{I}, s_{2} \cdots s_{p} p$ direzioni arbitrarie. Se ammettiamo la funzione $\varphi$ regolare, avremo che $\theta_{p}$ sarà una funzione regolare in tutto lo spazio, escluso il punto $r_{x}=0$. Seguiteremo a chiamare questo punto il centro delle onde sferiche, senonché lo diremo un centro multiplo di ordine $p$, per distinguere il caso in cui il potenziale di velocità è $\theta$, e in cui diremo che il punto $r_{\mathrm{I}}=\mathrm{o}$ è un centro semplice. Un centro multiplo è caratterizzato non solo dalla sua posizione, ma anche dalle $p$ direzioni $s_{I} s_{2} \cdots s_{p}$. In particolare un centro doppio sarà caratterizzato dalla sua posizione e dalla direzione $s_{x}$ secondo la quale si sono avvicinati indefinitamente i due centri semplici che lo costituiscono.

2. Nelia formula (2) distinguiamo la funzione arbitraria corrispondente al centro $x_{i} y_{i} z_{i}$, scrivendola

$$
\varphi_{i}\left(r_{i}-a t\right)=\varphi\left(x_{i}, y_{i}, z_{i}, r_{i}-a t\right) .
$$


Avremo allora

$$
\theta=\sum_{i}^{n} \frac{\varphi\left(x_{i}, y_{i}, z_{i}, r_{i}-a t\right)}{r_{i}} .
$$

Invece di considerare una somma come la precedente che corrisponde ad un numero finito di centri, consideriamo una espressione analoga corrispondente ad un sistema continuo di centri distribuiti in un certo spazio a tre dimensioni $\mathrm{S}$ o sopra una superficie $\sigma$ o una linea $s$. Otterremo in tal modo le funzioni

$$
\begin{aligned}
& \theta(x, y, z, t)=\int_{\mathrm{S}} \frac{\varphi(\xi, \eta, \zeta, r-a t)}{r} d \mathrm{~S} \\
& \theta(x, y, z, t)=\int_{\sigma} \frac{\varphi(\xi, \eta, \zeta, r-a t)}{r} d \sigma, \quad\left(r=\sqrt{(x-\xi)^{2}+(y-\eta)^{2}+(z-\zeta)^{2}}\right) \\
& \theta(x, y, z, t)=\int_{s} \frac{\varphi(\xi, \eta, \zeta, r-a t)}{r} d s,
\end{aligned}
$$

in cui $\xi, \eta, \zeta$ denotano rispettivamente le coordinate dei punti dello spazio $\mathrm{S}$, o della superflcie $\sigma$, o della linea $s$, di cui $d \mathrm{~S}, d \sigma, d s$ sono rispettivamente gli elementi di volume, d'area o di lunghezza.

Le funzioni così trovate soddisfaranno evidentemente, in tutti i punti esterni agli spazî $\mathrm{S}, \sigma, s$, la equazione differenziale (I), quando si ammetta che le funzioni $\varphi(\xi, \eta, \zeta, l)$ abbiano rispetto ad $l$ la derivata seconda finita e continua. Analogamente potremo ottenere infiniti altri integrali della equazione(I) considerando, invece delle distribuzioni continue di centri semplici, delle distribuzioni continue di centri multipli in modo che si abbia

$$
\theta(x, y, z, t)=\int_{\Sigma} \frac{d p}{d s_{1} d s_{2} \cdots d s_{p}}\left(\frac{\varphi(\xi, \eta, \zeta, r-a t)}{r}\right) d \Sigma
$$

in cui $\Sigma$ denota uno spazio a una, due o tre dimensioni. Si noti che in questa formula le derivate rispetto a $s_{1} s_{2} \cdots s_{p}$ vanno eseguite ritenendo $\varphi(\xi, \eta, \zeta, r$ - at $) / \gamma$ come funzione di $s_{1} s_{2} \cdots s_{p}$ per mezzo di $r$; ciò̀ le $\xi, \eta, \zeta$ che compariscono esplicitamente in $\varphi$ vanno considerate come costanti nell'eseguire le dette derivazioni.

La $\theta$ così ottenuta soddisfarà evidentemente l'equazione differenziale (I) in tutti i punti esterni allo spazio $\Sigma$.

3. Delle infinite funzioni che si possono costruire in questa guisa noi esamineremo in particolare le seguenti:

$\mathrm{I}^{\circ}$ le distribuzioni di centri semplici entro spazî a tre dimensioni; $2^{\circ}$ le distribuzioni di centri semplici sopra superficie;

$3^{\circ}$ le distribuzioni di centri doppî sopra superficie; essendo le direzioni corrispondenti ai centri doppî normali alle superficie stesse.

E evidente l'analogia fra la teoria che andiamo ora svolgendo e quella della funzione potenziale newtoniana. In questa ultima teoria si ha la funzione potenziale elementare $\mathrm{I} / \mathrm{r}$, e da essa si ricavano le funzioni potenziali 
dei corpi a tre dimensioni, delle superficie e dei doppî strati. Queste funzioni sono simili a quelle che si ottengono-dalle distribuzioni che vogliamo esaminare. Affinché queste ultime funzioni si riducano alle dette funzioni potenziali, basterà supporre che $\varphi(\xi, \eta, \zeta, l)$ sia indipendente da $l$. Allora $\varphi$ rappresenta la densità.

4. Cominciamo dall'estendere la formula di Poisson (Vedi Betti, Teorica delle forze newtoniane, Cap. I, $\S \mathrm{IX})$. A tal fine ammettiamo che la funzione $\varphi(\xi, \eta, \zeta, l)$ oltre ad avere la derivata seconda rispetto ad $l$ finita e continua goda rispetto a $\xi, \eta, \zeta$ delle proprietà richieste affinché considerata come una densità (cioè ritenendo $l$ costante) si possa applicare il teorema di PoIsson. Si ha

$$
\begin{aligned}
\theta & =\int_{S} \frac{\varphi(\xi, \eta, \zeta, r-a t)}{r} d \mathrm{~S}=\int_{\mathrm{S}} \frac{\varphi(\xi, \eta, \zeta,-a t)}{r} d \mathrm{~S} . \\
& +\int_{S} \frac{\varphi(\xi, \eta, \zeta, r-a t)-\varphi(\xi, \eta, \zeta,-a t)}{r} d \mathrm{~S} .
\end{aligned}
$$

Posto

$$
\theta_{\mathrm{x}}=\int_{\mathrm{S}} \frac{\varphi(\xi, \eta, \zeta,-a t)}{r} d \mathrm{~S} \quad, \quad \theta_{2}=\int_{\mathrm{S}} \frac{\varphi(\xi, \eta, \zeta, r-a t)-\varphi(\xi, \eta, \zeta,-a t)}{r} d \mathrm{~S}
$$

risulterà

$$
\theta=\theta_{\mathrm{r}}+\theta_{2} .
$$

Ora supponendo $t$ costante, $\theta_{x}$ è una ordinaria funzione potenziale, quindi per le condizioni poste, a cagione della formula di Poisson,

$$
\Delta_{2} \theta_{x}=-4 \pi \varphi(x, y, \dot{z},-a t)
$$

essendo $x, y, z$ un punto interno allo spazio $\mathrm{S}$.

Poniamo

$$
\frac{d \varphi}{d l}=\varphi_{1}(\xi, \eta, \zeta, l) \quad, \quad \frac{d^{2} \varphi}{d l^{2}}=\varphi_{2}(\xi, \eta, \zeta, l) .
$$

La funzione

$$
\mathrm{G}=\frac{\varphi(\xi, \eta, \zeta, r-a t)-\varphi(\xi, \eta, \zeta,-a t)}{r}=\varphi_{x}(\xi, \eta, \zeta, \vartheta r-a t)
$$

(in cui $\mathrm{I}>\vartheta>0$ ) $\grave{\mathrm{e}}$ finita e continua anche per $\xi=x, \eta=y, \zeta=z$. Derivandola si otterrà

$$
\begin{gathered}
\frac{\partial \mathrm{G}}{\partial x}=\left(\frac{\varphi_{1}(\xi, \eta, \zeta, r-a t)}{r}-\frac{\varphi(\xi, \eta, \zeta, r-a t)-\varphi(\xi, \eta, \zeta,-a t)}{r^{2}}\right) \frac{\partial r}{\partial x} \\
=\left(\frac{\varphi_{1}(\xi, \eta, \zeta, r-a t)-\varphi_{1}(\xi, \eta, \zeta, \vartheta r-a t)}{r}\right) \frac{\partial r}{\partial x} \\
=(1-\vartheta) \varphi_{2}\left(\xi, \eta, \zeta, \vartheta_{1} r-a t\right) \frac{\partial r}{\partial x} \quad\left(I>\vartheta_{1}\right.
\end{gathered}
$$

quindi $\hat{a} \mathrm{G} / \hat{\partial} x$ è finita anche per $\xi=x, \eta=y, \zeta=z$. 
Analogamente si vede che anche $\partial \mathrm{G} / \partial y, \partial \mathrm{G} / \partial z$ godono delle stesse proprietà. Derivando nuovamente la (3) avremo

$$
\begin{gathered}
\frac{\partial^{2} \mathrm{G}}{\partial x^{2}}=(1-\vartheta) \varphi_{2}\left(\xi, \eta, \zeta, \vartheta_{1} r-a t\right) \frac{\partial^{2} r}{\partial x^{2}}+\left(\frac{\varphi_{2}(\xi, \eta, \zeta, r-a t)}{r}-\frac{2 \varphi_{1}(\xi, \eta, \zeta, r-a t)}{r^{2}}\right. \\
\left.+2 \frac{\varphi(\xi, \eta, \zeta, r-a t)-\varphi(\xi, \eta, \zeta,-a t)}{r^{3}}\right)\left(\frac{\partial r}{\partial x}\right)^{2} \\
=(1-\vartheta) \varphi_{2}\left(\xi, \eta, \zeta, \vartheta_{1} r-a t\right) \frac{\partial^{2} r}{\partial x^{2}} \\
+\left(\frac{\varphi_{2}(\xi, \eta, \zeta, r-a t)-2(1-\vartheta) \varphi_{2}\left(\xi, \eta, \zeta, \vartheta_{1} r-a t\right)}{r}\right)\left(\frac{\partial r}{\partial x}\right)^{2}
\end{gathered}
$$

quindi $\partial^{2} \mathrm{G} / \partial x^{2}$, può tutto al più divenire infinita del $\mathrm{I}^{0}$ ordine per $\xi=x$, $\eta=y, \zeta=z$. Lo stesso può dirsi per $\partial^{2} \mathrm{G} / \partial y^{2}, \partial^{2} \mathrm{G} / \partial z^{2}$. Dalle formule precedenti segue che $\theta_{2}=\int \mathrm{G} d \mathrm{~S}$ e le sue derivate prime e seconde sono funzioni finite e continue, e che per calcolare il $\Delta^{2} \theta_{2}$, basta applicare la regola di derivazione sotto il segno. Si otterrà dunque

$$
\Delta^{2} \theta_{2}=\int_{\mathrm{S}}\left\{\frac{\mathrm{I}}{r} \Delta^{2} \varphi+2 \Delta\left(\varphi, \frac{\mathrm{I}}{r}\right)\right\} d \mathrm{~S}=\int_{\mathrm{S}}\left[\frac{\mathrm{I}}{r}\left(\frac{\partial^{2} \varphi}{\partial r^{2}} \Delta r+\frac{\partial \varphi}{\partial r} \Delta^{2} r\right)+2 \frac{\partial \varphi}{\partial r} \Delta\left(r, \frac{\mathrm{I}}{r}\right)\right] d \mathrm{~S} .
$$

$\mathrm{Ma}$

$$
\Delta r=\mathrm{I} \quad, \quad \Delta^{2} r=\frac{2}{r} \quad, \quad \Delta\left(r, \frac{\mathrm{I}}{r}\right)=-\frac{\mathrm{I}}{r^{2}}
$$

quindi

$$
\Delta^{2} \theta_{2}=\int_{S} \frac{1}{r} \frac{\partial^{2} \varphi}{\partial r^{2}} d S=\int_{S} \frac{1}{r} \frac{1}{a^{2}} \frac{\partial^{2} \varphi}{\partial t^{2}} d S=\frac{1}{a^{2}} \frac{\partial^{2}}{\partial t^{2}} \int_{S} \frac{\varphi}{r} d S=\frac{1}{a^{2}} \frac{\partial^{2} \theta}{\partial t^{2}} .
$$

$\mathrm{Ne}$ segue

$$
\Delta^{2} \theta=\Delta^{2} \theta_{x}+\Delta^{2} \theta_{2}=-4 \pi \varphi(x, y, z,-a t)+\frac{1}{a^{2}} \frac{\partial^{2} \theta}{\partial t^{2}}
$$

vale a dire

$$
\frac{\partial^{2} \theta}{\partial t^{2}}-a^{2} \Delta^{2} \theta=4 \pi a^{2} \varphi(x, y, z,-a t)
$$

in tutti i punti $x, y, z$ dello spazio $\mathrm{S}$.

In questa formula consiste la generalizzazione cercata della formula di PoIsson.

5. Passiamo ora ad esaminare una distribuzione superficiale di centri semplici. Ammettiamo la superficie senza punti singolari e supponiamo che $\varphi(\xi, \eta, \zeta, l)$ considerata come funzione di $\xi, \eta, \zeta$ ( $l$ ritenuto costante) goda delle proprietà che si attribuiscono alle densità superficiali nella teoria delle forze newtoniane (vedi BETTI, op. cit., $§$ VIII); supporremo inoltre, come precedentemente, che essa ammetta la derivata seconda rispetto ad $l$ finita e 
continua. Sarà quindi applicabile anche in questo caso la formula (3). Si avrà

$$
\begin{gathered}
\theta(x, y, z, t)=\int_{\sigma} \frac{\varphi(\xi, \eta, \zeta, r-a t)}{r} d \sigma= \\
=\int_{\sigma} \frac{\varphi(\xi, \eta, \zeta,-a t)}{r} d \sigma+\int_{\sigma} \frac{\varphi(\xi, \eta, \zeta, r-a t)-\varphi(\xi, \eta, \zeta,-a t)}{r} d \sigma .
\end{gathered}
$$

Posto

$$
\theta_{x}=\int_{\sigma} \frac{\varphi(\xi, \eta, \zeta,-a t)}{r} d \sigma \quad, \quad \theta_{2}=\int \frac{\varphi(\xi, \eta, \zeta, r-a t)-\varphi(\xi, \eta, \zeta,-a t)}{r} d \sigma
$$

potremo scrivere

$$
\theta=\theta_{\mathrm{r}}+\theta_{2} .
$$

Chiamiamo $n_{\mathrm{x}}$ e $n_{2}$ le normali alla superficie $\sigma$ dalle due parti di essa, le cui direzioni sono quelle secondo le quali ci si allontana dalla superficie stessa. Si avrà:

$$
\frac{\partial \theta_{1}}{\partial n_{x}}+\frac{\partial \theta_{x}}{\partial n_{2}}=-4 \pi \varphi(x, y, z,-a t) .
$$

La funzione $G$ che comparisce sotto al segno d'integrazione nella espressione di $\theta_{2}$ e le derivate $\partial \mathrm{G} / \partial x, \partial \mathrm{G} / \partial y, \partial \mathrm{G} / \partial z$ sono finite (vedi formula (3)) anche per $x=\xi, y=\eta, z=\zeta$; quindi $\theta_{2}$ e le sue derivate prime si mantengono finite e continue anche attraversando la superficie $\sigma$. Dalle (5) e (6) si deduce dunque:

$$
\frac{\partial \theta}{\partial n_{\mathrm{I}}}+\frac{\partial \theta}{\partial n_{2}}=-4 \pi \varphi(x, y, z,-a t) .
$$

(Cfr. la formula (6) del paragrafo citato del BETTI).

6. Consideriamo finalmente una distribuzione di centri doppî sopra una superficie $\sigma$, disposti normalmente ad essa. Avremo

$$
\begin{gathered}
\theta=\int_{\sigma} \frac{d}{d n} \frac{\varphi(\xi, \eta, \zeta, r-a t)}{r} d \sigma=\int_{\sigma} \varphi(\xi, \eta, \zeta, r-a t) \frac{\partial \frac{1}{r}}{\partial n} d \sigma+\int_{\sigma} \frac{1}{r} \frac{\partial \varphi}{\partial r} \frac{\partial r}{\partial n} d \sigma \\
=\int_{\sigma} \varphi(\xi, \eta, \zeta,-a t) \frac{\partial \frac{1}{r}}{\partial n} d \sigma \\
+\int_{\sigma}\left[\frac{\partial \varphi}{\partial r}-\frac{\varphi(\xi, \eta, \zeta, r-a t)-\varphi(\xi, \eta, \zeta,-a t)}{r}\right] \frac{1}{r} \frac{\partial r}{\partial n} d \sigma
\end{gathered}
$$

essendo $n$ normale a $\sigma$.

Posto

$$
\begin{gathered}
\theta_{\mathrm{I}}=\int_{\sigma} \varphi(\xi, \eta, \zeta,-a t) \frac{\partial \frac{\mathrm{I}}{r}}{\partial n} d \sigma \\
\theta_{2}=\int_{\sigma}\left[\frac{\partial \varphi}{\partial r}-\frac{\varphi(\xi, \eta, \zeta, r-a t)-\varphi(\xi, \eta, \zeta,-a t)}{r}\right] \frac{\mathrm{I}}{r} \frac{\partial r}{\partial n} d \sigma
\end{gathered}
$$


risulterà

$$
\theta=\theta_{1}+\theta_{2} .
$$

Ora $\theta_{1}$ (supponendo $t$ costante) è una funzione potenziale ordinaria di un doppio strato; quindi ammettendo che $\varphi(\xi, \eta, \zeta, l)$, considerata come funzione di $\xi, \eta, \zeta$, goda delle proprietà che si attribuiscono alle densità dei doppî strati (BETTI, op. cit., §XII), avremo

$$
\left(\theta_{x}\right)_{e}-\left(\theta_{x}\right)_{i}=4 \pi \varphi(x, y, z,-a t),
$$

denotando $\operatorname{con}\left(\theta_{x}\right)_{e}$ il valore che prende la funzione $\theta_{x}$ nel punto $x, y, z$ dalla parte di $\sigma$ da cui esce la direzione positiva di $n$ e con $\left(\theta_{I}\right)_{i}$ il valore di $\theta_{x}$ nello stesso punto $x, y, z$, ma dalla parte di $\sigma$ da cui entra la direzione positiva di $n$; mentre

$$
\left(\frac{\partial \theta_{I}}{\partial n}\right)_{e}=\left(\frac{\partial \theta_{x}}{\partial n}\right)_{i}
$$

Avremo poi che la funzione

$$
\mathrm{H}=\left[\frac{\partial \varphi}{\partial r}-\frac{\varphi(\xi, \eta, \zeta, r-a t)-\varphi(\xi, \eta, \zeta,-a t)}{r}\right] \frac{\mathrm{I}}{r} \frac{\partial r}{\partial n}
$$

è finita anche per $x=\xi, y=\eta, z=\zeta$. Infatti può scriversi

$$
\begin{gathered}
H=\left[\varphi_{1}(\xi, \eta, \zeta, r-a t)-\varphi_{1}(\xi, \eta, \zeta, \vartheta r-a t)\right] \frac{1}{r} \frac{\partial r}{\partial n} \\
=(1-\vartheta) \varphi_{2}\left(\xi, \eta, \zeta, \vartheta_{1} r-a t\right) \frac{\partial r}{\partial n} .
\end{gathered}
$$

Ne segue che $\theta_{2}=\int_{\sigma} H d \sigma$ si conserverà continua anche attraversando la superficie $\sigma$.

Avremo poi

$$
\begin{gathered}
\frac{\partial \mathrm{H}}{\partial x}=-\left[\frac{\partial \varphi}{\partial r}-\frac{\varphi(\xi, \eta, \zeta, r-a t)-\varphi(\xi, \eta, \zeta,-a t)}{r}\right] \frac{\mathrm{I}}{r^{2}} \frac{\partial r}{\partial x} \frac{\partial r}{\partial n} \\
+\left[\frac{\partial^{2} \varphi}{\partial r^{2}}-\frac{\mathrm{I}}{r} \frac{\partial \varphi}{\partial r}+\frac{\varphi(\xi, \eta, \zeta, r-a t)-\varphi(\xi, \eta, \zeta,-a t)}{r^{2}}\right] \frac{\mathrm{I}}{r} \frac{\partial r}{\partial x} \frac{\partial r}{\partial n} \\
+\left[\frac{\partial \varphi}{\partial r}-\frac{\varphi(\xi, \eta, \zeta, r-a t)-\varphi(\xi, \eta, \zeta,-a t)}{r}\right] \frac{\mathrm{I}}{r} \frac{\partial}{\partial x} \frac{\partial r}{\partial n} \\
=\left[-2(\mathrm{I}-\vartheta) \varphi_{2}\left(\xi, \eta, \zeta, \vartheta_{1} r-a t\right)+\varphi_{2}(\xi, \eta, \zeta, r-a t)\right] \frac{\mathrm{I}}{r} \frac{\partial r}{\partial x} \frac{\partial r}{\partial n} \\
+(\mathrm{I}-\vartheta) \varphi_{2}\left(\zeta, \eta, \zeta, \vartheta_{1} r-a t\right) \frac{\partial}{\partial x} \frac{\partial r}{\partial n} .
\end{gathered}
$$

Quindi $\partial \mathrm{H} / \partial x$ potrà al più divenire infinita del $\mathrm{I}^{\circ}$ ordine per $x=\xi$, $y=\eta, z=\zeta$. Lo stesso vale evidentemente per $\partial \mathrm{H} / \partial y, \partial \mathrm{H} / \partial z$. Se ne conclude che anche le derivate prime di $\theta_{2}$ si manterranno continue attraversando la superficie $\sigma$. Dunque

$$
\left(\theta_{2}\right)_{e}-\left(\theta_{2}\right)_{i}=0 \quad, \quad\left(\frac{\partial \theta_{2}}{\partial n}\right)_{e}-\left(\frac{\partial \theta_{2}}{\partial n}\right)_{i}=0
$$


e per conseguenza, tenendo presenti le (8), (9),

$$
\left\{\begin{array}{l}
(\theta)_{e}-(\theta)_{i}=4 \pi \varphi(\xi, \eta, \zeta,-a t) \\
\left(\frac{\partial \theta}{\partial n}\right)_{e}-\left(\frac{\partial \theta}{\partial n}\right)_{i}=0 .
\end{array}\right.
$$

7. Veduta così mediante le formule (4) (7) (I0) la estensione della teoria ordinaria della funzione potenziale alle distribuzioni continue di centri semplici e doppî, ricordiamo che la formula di GREEN conduce immediatamente al teorema seguente: Ogni funzione armonica ${ }^{(\mathrm{I})}$ regolare entro uno spazio $s$ può ritenersi come la funzione potenziale di una superficie e di un doppio strato distribuiti sul contorno $\sigma$.

Infatti se $\mathrm{V}$ è una funzione armonica regolare entro $\mathrm{S}$, la formula di Green dà (Vedi BetTI, op. cit., § XI form. (8))

$$
\mathrm{V}(x, y, z)=\frac{\mathrm{I}}{4 \pi} \int_{\sigma}\left(\mathrm{V} \frac{d \frac{\mathrm{I}}{r}}{d n}-\frac{d \mathrm{~V}}{d n} \frac{\mathrm{I}}{r}\right) d \sigma
$$

in cui $r=\sqrt{(x-\xi)^{2}+(y-\eta)^{2}+(z-\zeta)^{2}}$ (essendo $\xi, \eta, \zeta$ il punto variabile sopra la superficie $\sigma$ ) e in cui $n$ è la normale (interna ad S) alla superficie stessa. Ponendo

$$
\frac{\mathrm{V}(\xi, \eta, \zeta)}{4 \pi}=f(\xi, \eta, \zeta) \quad, \quad-\frac{\mathrm{I}}{4 \pi} \frac{d \mathrm{~V}}{d n}=\varphi(\xi, \eta, \zeta)
$$

la formula precedente diviene

$$
\mathrm{V}(x, y, z)=\int_{\sigma} f(\xi, \eta, \zeta) \frac{d \frac{\mathrm{I}}{r}}{d n} d \sigma+\int_{\sigma} \varphi(\xi, \eta, \zeta) \frac{\mathrm{I}}{r} d \sigma
$$

la quale mostra chiaramente il significato che abbiamo attribuito al teorema di GREEN.

Sovrapponiamo ora nel contorno $\sigma$ dello spazio $\mathrm{S}$ due distribuzioni di centri semplici e di centri doppî normali a $\sigma$. Otterremo in tal modo

$$
\theta(x, y, z, t)=\int_{\sigma} \frac{\varphi(\xi, \eta, \zeta, r-a t)}{r} d \sigma+\int_{\sigma} \frac{d}{d n} \frac{f(\xi, \eta, \zeta, r-a t)}{r} d \sigma .
$$

La funzione $\theta(x, y, z, t)$ soddisfa evidentemente in tutti i punti interni ad S l'equazione(I). Proponiamoci ora di vedere se il teorema precedentemente enunciato per le funzioni armoniche può estendersi agli integrali regolari della (I), cioè se tutte le funzioni regolari entro $\mathrm{S}$ che soddisfano la (I) possono porsi sotto la forma (I2).

Per ottenere questa estensione seguiremo un procedimento analogo a quello col quale nella teoria della funzione potenziale si giunge alla formula (I I) partendo dal iemma di GreEN.

(I) Per funzione armonica intendiamo una funzione che soddisfa la equazione differenziale $\Delta^{2}=0$. 
Se $\psi$ e $\chi$ sono due funzioni regolari entro il campo S, limitato dal contorno $\sigma$, il detto lemma ci dà

$$
\int_{\sigma}\left(\frac{\partial \psi}{\partial n} \chi-\frac{\partial \chi}{\partial n} \psi\right) d \sigma=\int_{\mathrm{S}}\left(\psi \Delta^{2} \chi-\chi \Delta^{2} \psi\right) d \mathrm{~S} .
$$

Supponiamo ora che si abbia

$\frac{\partial^{2} \psi}{\partial t^{2}}-a^{2} \Delta^{2} \psi+\Psi(x, y, z, t)=0 \quad, \quad \frac{\partial^{2} \chi}{\partial t^{2}}-a^{2} \Delta^{2} \chi+\mathrm{X}(x, y, z, t)=0 ;$

allora l'equazione precedente diviene

$$
\begin{gathered}
\int_{\sigma}\left(\frac{\partial \psi}{\partial n} \chi-\frac{\partial \chi}{\partial n} \psi\right) d \sigma=\frac{1}{a^{2}} \int_{\mathrm{S}}\left(\frac{\partial^{2} \chi}{\partial t^{2}} \psi-\frac{\partial^{2} \psi}{\partial t^{2}} \chi\right) d \mathrm{~S}-\frac{1}{a^{2}} \int_{\mathrm{S}}(\Psi \chi-\mathrm{X} \psi) d \mathrm{~S} \\
=\frac{1}{a^{2}} \frac{\partial}{\partial t} \int_{\mathrm{S}}\left(\frac{\partial \chi}{\partial t} \psi-\frac{\partial \psi}{\partial t} \chi\right) d \mathrm{~S}-\frac{\mathrm{I}}{a^{2}} \int_{\mathrm{S}}(\Psi \chi-\mathrm{X} \psi) d \mathrm{~S}
\end{gathered}
$$

Se prendiamo $\chi=f(r+a t) / r$, ove con $r$ si denotano le distanze contate da un punto fisso $x_{0}, y_{0}, z_{0}$ interno allo spazio $\mathrm{S}$, questa funzione non sarà regolare nel punto $x_{0}, y_{0}, z_{0}$ stesso, mentre se $f$ è una funzione regolare, tale sarà $\chi$ in tutti gli altri punti di S. Volendo dunque applicare la (13) bisognerà escludere il detto punto $x_{0}, y_{0}, z_{0}$. Faremo questo mediante una sfera $\omega$ avente il centro in $x_{0}, y_{0}, z_{0}$ ed il raggio $\mathrm{R}$. Chiamando quindi $\mathrm{S}^{\prime}$ lo spazio $\mathrm{S}$ da cui si sia tolto lo spazio racchiuso entro la sfera $\omega$, si avrà, osservando che $\mathrm{X}=\mathrm{o}$,

$$
\begin{gathered}
\int_{\sigma}\left[\frac{\partial \psi}{\partial n} \frac{f(r+a t)}{r}-\frac{\partial}{\partial n}\left(\frac{f(r+a t)}{r}\right) \psi\right] d \sigma \\
+\int_{\omega}\left[\frac{\partial \psi}{\partial n} \frac{f(r+a t)}{r}-\frac{\partial}{\partial n}\left(\frac{f(r+a t)}{r}\right) \psi\right] d \omega+\frac{\mathrm{I}}{a^{2}} \int_{\mathrm{S}^{\prime}} \Psi \frac{f(r+a t)}{r} d \mathrm{~S}^{\prime} \\
=\frac{\mathrm{I}}{a^{2}} \frac{\partial}{\partial t} \int_{\mathbf{S}^{\prime}}\left[\frac{\partial}{\partial t}\left(\frac{f(r+a t)}{r}\right) \psi-\frac{\partial \psi}{\partial t} \frac{f(r+a t)}{r}\right] d \mathrm{~S}^{\prime} .
\end{gathered}
$$

Consideriamo ora in particölare il secondo integrale del primo membro. Chiamando $d \alpha$ l'elemento della sfera di raggio $\mathrm{I}$, avremo

$$
d \omega=\mathrm{R}^{2} d \alpha,
$$

quindi il detto integrale potrà scriversi

$$
\begin{gathered}
\mathrm{J}=\int_{\alpha}\left[\frac{\partial \psi}{\partial n} \frac{f(\mathrm{R}+a t)}{\mathrm{R}}-\psi \frac{f^{\prime}(\mathrm{R}+a t)}{\mathrm{R}}+\psi \frac{f(\mathrm{R}+a t)}{\mathrm{R}^{2}}\right] \mathrm{R}^{2} d \alpha \\
=\mathrm{R} \int_{\alpha}\left[\frac{\partial \psi}{\partial n} f(\mathrm{R}+a t)-\psi f^{\prime}(\mathrm{R}+a t)\right] d \alpha+\int_{\alpha} \psi f(\mathrm{R}+a t) d \alpha
\end{gathered}
$$

onde facendo impiccolire indefinitamente $\mathrm{R}$,

$$
\lim _{\mathrm{R}=\mathrm{o}} \mathrm{J}=4 \pi \psi\left(x_{0}, y_{0}, z_{0}, t\right) f(a t) \text {. }
$$


La formula (14) potrà dunque scriversi, osservando che l'ultimo integraie del primo membro è proprio,

$$
\begin{gathered}
4 \pi \psi\left(x_{0}, y_{0}, z_{0}, t\right) f(a t)+\int_{\sigma}\left[\frac{\partial \psi}{\partial n} \frac{f(r+a t)}{r}-\frac{\partial}{\partial n}\left(\frac{f(r+a t)}{r}\right) \psi\right] d \sigma \\
+\frac{\mathrm{I}}{a^{2}} \int_{\mathrm{S}} \Psi \frac{f(r+a t)}{r} d \mathrm{~S}=\frac{\mathrm{I}}{a^{2}} \frac{\partial}{\partial t} \int\left[\frac{\partial}{\partial t}\left(\frac{f(r+a t)}{r}\right) \psi-\frac{\partial \psi}{\partial t} \frac{f(r+a t)}{r}\right] d \mathrm{~S} .
\end{gathered}
$$

Abbiamo ora

$$
\begin{gathered}
\psi \frac{\partial}{\partial n}\left(\frac{f(r+a t)}{r}\right)=\psi \frac{\partial \frac{1}{r}}{\partial n} f(r+a t)+\frac{1}{r} \psi f^{\prime}(r+a t) \frac{\partial r}{\partial n} \\
=\psi \frac{\partial \frac{1}{r}}{\partial n} f(r+a t)-\frac{1}{a r} \frac{\partial \psi}{\partial t} f(r+a t) \frac{\partial r}{\partial n}+\frac{1}{a} \frac{\partial}{\partial t}\left(\frac{1}{r} \psi f(r+a t) \frac{\partial r}{\partial n}\right)
\end{gathered}
$$

quindi

$$
\begin{aligned}
& 4 \pi \psi\left(x_{0}, y_{0}, z_{0}, t\right) f(a t)+\int_{\sigma}\left[\frac{\partial \psi}{\partial n} \frac{\mathrm{I}}{r}-\psi \frac{\partial \frac{\mathrm{I}}{r}}{\partial n}+\frac{\mathrm{I}}{a r} \frac{\partial \psi}{\partial t} \frac{\partial r}{\partial n}\right] f(r+a t) d \sigma \\
&+\frac{\mathrm{I}}{a^{2}} \int_{\mathrm{S}} \Psi \frac{\mathrm{I}}{r} f(r+a t) d \mathrm{~S}=\frac{\partial}{\partial t}\left\{\frac{\mathrm{I}}{a^{2}} \int\left[\frac{\partial}{\partial t}\left(\frac{f(r+a t)}{r}\right) \psi-\frac{\partial \psi}{\partial t} \frac{f(r+a t)}{r}\right] d \mathrm{~S}\right. \\
&\left.+\frac{\mathrm{I}}{a} \int_{\sigma} \frac{\mathrm{I}}{r} \psi f(r+a t) \frac{\partial r}{\partial n} d \sigma\right\}
\end{aligned}
$$

Suppongasi che la funzione $f(x)$ che per ora abbiamo lasciata indeterminata si annulli per tutti i valori dell'argomento superiori ad un certo limite $\mathrm{N}$ e per tutti quelli inferiori al limite $-\mathrm{N}$; allora moltiplicando ambo i membri della equazione precedente per $d t$ e integrando fra $-\infty$ e $+\infty$, il secondo membro andrà a zero, e per conseguenza otterremo

$$
\int_{-\infty}^{\infty} 4 \pi \psi\left(x_{\circ}, y_{0}, z_{0}, t\right) f(a t) d t
$$

$+\int_{-\infty}^{\infty} d t \int\left[\frac{\partial \psi}{\partial n} \frac{\mathrm{I}}{r}-\psi \frac{\partial \frac{\mathrm{I}}{r}}{\partial n}+\frac{\mathrm{I}}{a r} \frac{\partial \psi}{\partial t} \frac{\partial r}{\partial n}\right] f(r+a t) d \sigma+\int_{-\infty}^{\infty} \frac{d t}{a^{2}} \int_{\mathrm{S}} \Psi \frac{\mathrm{I}}{r} f(r+a t) d \mathrm{~S}=0$.

Denotiamo con $\xi, \eta, \zeta$, le coordinate dei punti del contorno $\sigma$ e con $x, y, z$ quelle dei punti di S. Le quantità che compariscono nei due ultimi integrali saranno

$$
\begin{gathered}
\frac{\partial \psi}{\partial n}=\frac{\partial \psi}{\partial \xi} \frac{\partial \xi}{\partial n}+\frac{\partial \psi}{\partial \eta} \frac{\partial \eta}{\partial n}+\frac{\partial \psi}{\partial \zeta} \frac{\partial \zeta}{\partial n}=\psi_{I}(\xi, \eta, \zeta, t) \\
\psi=\psi(\xi, \eta, \zeta, t) \\
\frac{\partial \psi}{\partial t}=\frac{\partial \psi(\xi, \eta, \zeta, t)}{\partial t}=\psi_{2}(\xi, \eta, \zeta, t) \\
\Psi=\Psi(x, y, z, t)
\end{gathered}
$$


onde se nei due detti integrali poniamo in luogo di $t, t-\frac{r}{a}$, la loro somma diverrà

$$
\begin{aligned}
& \mathrm{K}=\int_{-\infty}^{\infty} d t \int_{\sigma}\left\{\psi_{\mathrm{I}}\left(\xi, \eta, \zeta, t-\frac{r}{a}\right) \frac{\mathrm{I}}{r}-\psi\left(\xi, \eta, \zeta, t-\frac{r}{a}\right) \frac{\partial \frac{\mathrm{I}}{r}}{\partial n}\right. \\
&\left.+\frac{\mathrm{I}}{a r} \psi_{2}\left(\xi, \eta, \zeta, t-\frac{r}{a}\right) \frac{\partial r}{\partial n}\right\} f(a t) d \sigma+\int_{-\infty}^{\infty} d t \int_{\mathrm{S}} \frac{\Psi\left(x, y, z, t-\frac{r}{a}\right)}{a^{2} r} f(a t) d \mathrm{~S} \\
&=\int_{-\infty}^{\infty} f(a t) d t\left\{\int _ { \sigma } ^ { \infty } \left[\psi_{\mathrm{I}}\left(\xi, \eta, \zeta, t-\frac{r}{a}\right) \frac{\mathrm{I}}{r}-\psi\left(\xi, \eta, \zeta, t-\frac{r}{a}\right) \frac{\partial \frac{\mathrm{I}}{r}}{\partial n}\right.\right. \\
&\left.\left.-\frac{\mathrm{I}}{r} \frac{\partial \psi\left(\xi, \eta, \zeta, t-\frac{r}{a}\right)}{\partial r} \frac{\partial r}{\partial n}\right] d \sigma+\frac{\mathrm{I}}{a^{2}} \int_{\mathrm{S}}^{\Psi} \frac{\Psi\left(x, y, z, t-\frac{r}{a}\right)}{r} d \mathrm{~S}\right\}
\end{aligned}
$$

Ora se rappresentiamo con $\frac{\partial}{\partial n}\left[\frac{\psi\left(\xi, \eta, \zeta, t-\frac{r}{a}\right)}{r}\right]$ la derivata presa rispetto ad $n$ del rapporto $\frac{\psi\left(\xi, \eta, \zeta, t-\frac{r}{a}\right)}{r}$, ritenendo le $\xi, \eta, \zeta$, che compariscono esplicitamente, come costanti e la sola $r$ funzione di $n$ (Cfr. il $\S 2$ ) avremo

$$
\frac{\partial}{\partial n}\left[\frac{\psi\left(\xi, \eta, \zeta, t-\frac{r}{a}\right)}{r}\right]=\psi\left(\xi, \eta, \zeta, t-\frac{r}{a}\right) \frac{\partial \frac{1}{r}}{\partial n}+\frac{1}{r} \frac{\partial \psi\left(\xi, \eta, \zeta, t-\frac{r}{a}\right)}{\partial r} \frac{\partial r}{\partial n}
$$

$\psi\left(\xi, \eta, \zeta, t-\frac{r}{a}\right)$ rappresenta invece la derivata di $\psi\left(\xi, \eta, \zeta, t-\frac{r}{a}\right)$ rispetto ad $n$, ritenendo $r$ costante e soltanto le $\xi, \eta, \zeta$ che compariscono esplicitamente come funzioni di $n$ [(Vedi formula (I 7)]. Per distinguere la derivata rispetto ad $n$ presa in questa ipotesi, da quella che abbiamo indicata precedentemente col simbolo $\partial / \partial n$, la denoteremo col simbolo $\delta / \delta n$. Per conseguenza

$$
\begin{gathered}
\mathrm{K}=\int_{-\infty} f(a t) d t\left\{\int_{\sigma}\left(\frac{\delta}{\delta n}\left[\frac{\psi\left(\xi, \eta, \zeta, t-\frac{r}{a}\right)}{r}\right]-\frac{\partial}{\partial n}\left[\frac{\psi\left(\xi, \eta, \zeta, t-\frac{r}{a}\right)}{r}\right]\right) d \sigma\right. \\
\left.+\frac{\mathrm{I}}{a^{2}} \int_{\mathrm{S}}^{\infty} \frac{\Psi\left(x, y, z, t-\frac{r}{a}\right)}{r} d \mathrm{~S}\right\}
\end{gathered}
$$

e la equazione (I6) potrà scriversi

$$
\int_{-\infty}^{\infty} f(a t) d t\left\{4 \pi \psi\left(x_{0}, y_{0}, z_{0}, t\right)+\int_{\sigma}\left(\frac{\delta}{\delta n}\left[\frac{\psi\left(\xi, \eta, \zeta, t-\frac{r}{a}\right)}{r}\right]-\right.\right.
$$




$$
\left.\left.-\frac{\partial}{\partial n}\left[\frac{\psi\left(\xi, \eta, \zeta, t-\frac{r}{a}\right)}{r}\right]\right) d \sigma+\frac{\mathrm{I}}{a^{2}} \int_{\mathrm{S}} \frac{\Psi\left(x, y, z, t-\frac{r}{a}\right)}{r} d \mathrm{~S}\right\}=0
$$

Ora $f(a t)$ è una funzione arbitraria. Affinchè dunque la formula precedente possa sussistere, bisognerà che si abbia

$$
\begin{aligned}
& 4 \pi \psi\left(x_{0}, y_{0}, z_{0}, t\right)+\int_{\sigma}^{1}\left\{\frac{\delta}{\delta n}\left(\frac{\psi\left(\xi, \eta, \zeta, t-\frac{r}{a}\right)}{r}\right)-\frac{\partial}{\partial n}\left(\frac{\psi\left(\xi, \eta, \zeta, t-\frac{r}{a}\right)}{r}\right)\right\} d \sigma
\end{aligned}
$$

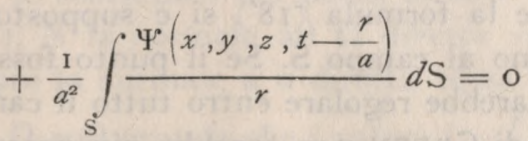

d'onde

$$
\begin{gathered}
\psi\left(x_{0}, y_{0}, z_{0}, t\right)=\frac{1}{4 \pi} \int_{\sigma}\left\{\frac{\partial}{\partial n}\left(\frac{\psi\left(\xi, \eta, \zeta, t-\frac{r}{a}\right)}{r}\right)\right. \\
\left.-\frac{\delta}{\delta n}\left(\frac{\psi\left(\xi, \eta, \zeta, t-\frac{r}{a}\right)}{r}\right)\right\} d \sigma-\frac{1}{4 \pi a^{2}} \int_{\mathrm{S}} \frac{\Psi\left(x, y, z, t-\frac{r}{a}\right)}{r} d \mathrm{~S} .
\end{gathered}
$$

Questa formula è dovuta al BeLtrami. Supponendo che $\psi$ sia un integrale della equazione

$$
\frac{\partial^{2} \theta}{\partial t^{2}}=a^{2} \Delta^{2} \theta
$$

$\Psi$ si riduce eguale a zero, e si ottiene la formula di KIRCHHOFF

$\left(\mathrm{I} 8^{\prime}\right) \psi(x, y, z, t)=\frac{\mathrm{I}}{4 \pi} \int_{\sigma}\left\{\frac{\partial}{\partial n}\left(\frac{\psi\left(\xi, \eta, \zeta, t-\frac{r}{a}\right)}{r}\right)-\frac{\delta}{\delta n}\left(\frac{\psi\left(\xi, \eta, \zeta, t-\frac{r}{a}\right)}{r}\right)\right\} d \sigma$ in cui si è posto $x, y, z$ in luogo di $x_{0}, y_{0}, z_{0}$ che compariscono nella formula precedente.

Essa è appunto la generalizzazione della formula (I I) che cercavamo. Si ponga

$$
\begin{gathered}
\frac{1}{4 \pi} \psi\left(\xi, \eta, \zeta, t-\frac{r}{a}\right)=\varphi(\xi, \eta, \zeta, r-a t), \\
-\frac{1}{4 \pi} \frac{\delta \psi\left(\xi, \eta, \zeta, t-\frac{r}{a}\right)}{\delta n}=-\frac{1}{4 \pi} \psi_{x}\left(\xi, \eta, \zeta, t-\frac{r}{a}\right)=f(\xi, \eta, \zeta, r-a t)
\end{gathered}
$$

la $\left(\right.$ I $\left.^{\prime}\right) \cdot$ potrà scriversi

(I9) $\psi(x, y, z, t)=\int_{\sigma}\left\{\frac{\partial}{\partial n}\left(\frac{\varphi(\xi, \eta, \zeta, r-a t)}{r}\right)+\frac{f(\xi, \eta, \zeta, r-a t)}{r}\right\} d \sigma$. 
Avremo quindi il teorema:

Ogni funzione $\psi(x, y, z, t)$ regolare entro un campo finito $\mathrm{S}$ e che soddisfa l'equazione differenziale

$$
\frac{\partial^{2} \psi}{\partial t^{2}}=a^{2} \Delta^{2} \psi
$$

potrà considerarsi come il potenziale di velocità (o come il potenziale degli spostamenti) dovuto a due distribuzioni sopra il contorno $\sigma$ : l'una di centri semplici, l'altra di centri doppî normali a $\sigma$. stesso.

8. Nello stabilire la formula ( $\left.18^{\prime}\right)$ si è supposto che il punto $x, y, z$ fosse un punto interno al campo S. Se il punto fosse esterno al campo la funzione $f(r+a t) / r$ sarebbe regolare entro tutto il campo stesso; quindi nell'applicare il lemma di GREEN non sarebbe più necessario escludere alcuna porzione di S. Nella (14) mancherebbe dunque l'integrale esteso ad $\omega$ e perciò si giungerebbe alla formula

$$
\mathrm{O}=\frac{\mathrm{I}}{4 \pi} \int_{\sigma}\left\{\frac{\partial}{\partial n}\left[\frac{\psi\left(\xi, \eta, \zeta, t-\frac{r}{a}\right)}{r}\right]-\frac{\delta \psi\left(\xi, \eta, \zeta, t-\frac{r}{a}\right)}{\delta n} \frac{\mathrm{I}}{r}\right\} d \sigma .
$$

Finalmente se il punto $x, y, z$ facesse parte del contorno $\sigma$, nella (I4) dovrebbe limitarsi il secondo integrale che comparisce nel primo membro alla porzione della sfera $\omega$ che giace nell'interno di $\mathrm{S}$ onde alla equazione (I 5 ) dovrebbe sostituirsi l'altra

$$
\lim _{\mathrm{R}=\mathrm{o}} \mathrm{J}=v \psi(x, y, z, t) f(a t),
$$

essendo $v$ ciò che si suol chiamare l'angolo visuale del contorno $\sigma$ relativo al punto $x, y, z$. Quindi in questo caso invece della ( $\left.18^{\prime}\right)$ avremo la formula

$$
v \psi(x, y, z, t)=\int_{\sigma}\left\{\frac{\partial}{\partial n}\left[\frac{\psi\left(\xi, n, \zeta, t-\frac{r}{a}\right)}{r}\right]-\frac{\delta \psi\left(\xi, \eta, \zeta, t-\frac{r}{a}\right)}{\delta n} \frac{1}{r}\right\} d \sigma .
$$

Questa formula comprende le due precedenti ( $\left.18^{\prime}\right)$ e ( $\left.18^{\prime \prime}\right)$ quando si ritenga (come si suol fare ordinariamente) che l'angolo visuale di $\sigma$ rispetto ad un punto interno sia $4 \pi$, e rispetto ad un punto esterno sia zero.

9. Le formule trovate valgono quando $\sigma$ sia un contorno formato da una o da più superficie finite che limitano lo spazio finito $S$ entro il quale la funzione $\psi$ è regolare e soddisfa la (I). Supponiamo ora che lo spazio $\mathrm{S}$ entro il quale la funzione $\psi$ è regolare e soddisfa la (I), si estenda all'infinito e sia limitato a distanza finita da una o da più superficie chiuse il cui insieme denoteremo sempre con $\sigma$. Ammettiamo inoltre che la funzione $\psi(x, y, z, t)$ per tutti i valori di $t$ inferiori ad un certo limite $\mathrm{T}$ sia sempre nulla. $\mathrm{Si}$ conduca col centro nell'origine una sfera $\Omega$ di raggio $\mathrm{R}$ la quale includa tutto 
l'insieme di superficie $\sigma$ e il punto $x, y, z$, e consideriamo lo spazio $\mathrm{S}^{\prime}$ limitato da $\sigma$ e da $\Omega$. Applicando la formula (I $8^{\prime}$ ) allo spazio $S^{\prime}$ avremo

$$
\begin{gathered}
\psi(x, y, z, t)=\frac{\mathrm{I}}{4 \pi} \int_{\sigma}\left\{\frac{\partial}{\partial n}\left[\frac{\psi\left(\xi, \eta, \zeta, t-\frac{r}{a}\right)}{r}\right]-\frac{\delta}{\delta n} \psi\left(\xi, \eta, \zeta, t-\frac{r}{a}\right) \cdot \frac{\mathrm{I}}{r}\right\} d \sigma \\
\quad+\frac{\mathrm{I}}{4 \pi} \int_{\Omega}\left\{\frac{\partial}{\partial \mathrm{N}}\left[\frac{\psi\left(\xi^{\prime}, \eta^{\prime}, \zeta^{\prime}, t-\frac{r^{\prime}}{a}\right)}{r^{\prime}}\right]-\frac{\delta}{\delta n} \psi\left(\xi^{\prime}, \eta^{\prime}, \zeta^{\prime}, t-\frac{r^{\prime}}{a}\right) \cdot \frac{\mathrm{I}}{r^{\prime}}\right\} d \Omega
\end{gathered}
$$

chiamando $\xi^{\prime}, \eta^{\prime}, \zeta^{\prime}$ le coordinate dei punti di $\Omega ; r^{\prime}$ le distanze dei punti di $\Omega$ dal punto $x, y, z ; \mathrm{N}$ la normale ad $\Omega$ diretta verso l'interno di $\mathrm{S}^{\prime}$. Con $n$ si intenderà sempre la normale a $\sigma$ diretta verso l'interno di S. Si prenda il raggio della sfera $\Omega$ così grande che i valori $t-\frac{r^{\prime}}{a}$ risultino tutti inferiori a T. Avremo allora che il secondo integrale contenuto nel secondo membro della formula precedente sparirà, onde la formula $\left(18^{\prime}\right)$ si potrà applicare anche nel caso in cui lo spazio S si estenda all'infinito. Il teorema precedente varrà dunque anche nel caso di uno spazio infinito limitato a distanza finita da una o da più superficie chiuse, quando si aggiunga la condizione che $\psi$ si annulli per valori di $t$ inferiori ad un certo limite.

Ammettendo sempre soddisfatte le condizioni stabilite in questo teorema, sussisterà anche la formula ( $\left.18^{\prime \prime}\right)$ quando il punto $x, y, z$ sia esterno allo spazio $\mathrm{S}$, ed in generale potremo scrivere la formula (20) considerando come contorno completo dello spazio S, l'insieme di superficie $\sigma$ e la sfera all'infinito.

IO. Sia $\psi(x, y, z, t)$ una funzione regolare di $x, y, z, t$ in tutto lo spazio la quale si annulli per tutti i valori di $t$ inferiori ad un certo limite e soddisfi l'equazione differenziale (I). Consideriamo un punto qualunque $x, y, z$ esterno ad una superficie chiusa $\sigma$. A cagione della ( $18^{\prime \prime}$ ) avremo

$$
\mathrm{O}=\frac{\mathrm{I}}{4 \pi} \int_{\sigma}\left\{\frac{d}{d n}\left[\frac{\psi\left(\xi, \eta, \zeta, t-\frac{r}{a}\right)}{r}\right]-\frac{\delta}{\delta n} \psi\left(\xi, \eta, \zeta, t-\frac{r}{a}\right) \cdot \frac{1}{r}\right\} d \sigma .
$$

$\mathrm{Ma}$, essendo $\psi$ regolare anche nello spazio esterno a $\sigma$ ed annullandosi per valori di $t$ inferiori ad un certo limite, il secondo membro della formula precedente esprimerà il valore di $\psi(x, y, z, t)$. Se ne conclude che la funzione $\psi$ è sempre eguale a zero. Quindi si avrà il teorema: una funzione regolare in tutto lo spazio che si annulla per valori di t inferiori ad un certo limite e soddisfa la (I) è sempre nulla.

II. In tutto ciò che segue ammetteremo sempre soddisfatta la condizione che le funzioni $\psi(x, y, z, t)$ che si considerano si annullino per valori di $t$ inferiori ad un certo limite, anche quando non diremo questa condizione esplicitamente. 
Ciò premesso i teoremi dei paragrafi precedenti 7,9 potranno enunciarsi nel modo seguente.

Abbiasi il moto di un fluido dovuto ad onde emananti da un sistema qualunque di centri. Per esaminare il moto in un punto qualunque potrà sempre sostituirsi ai centri dati un sistema di altri centri distribuiti sopra una superficie, o un sistema di superficie $\sigma$ qualunque che separi $i$ centri dati dal punto in cui si vuol studiare il moto.

Gli elementi relativi al moto (potenziale di velocità, potenziale degli spostamenti) in un dato istante nel punto che si considera dipenderanno dagli elementi relativi al movimento (potenziale degli spostamenti, di velocità, ecc.) nei punti delle dette superficie $\sigma$ in istanti anteriori. Per precisare tale dipendenza riprendiamo la formula di KIRCHHOFF sotto la forma

$$
\begin{aligned}
& \psi(x, y, z, t)=\frac{1}{4 \pi} \int_{\sigma}\left\{-\psi_{2}\left(\xi, \eta, \zeta, t-\frac{r}{a}\right) \frac{\mathrm{I}}{a r} \frac{\partial r}{\partial n}\right. \\
& \left.+\psi\left(\xi, \eta, \zeta, t-\frac{r}{a}\right) \frac{\partial \frac{\mathrm{I}}{r}}{\partial n}-\psi_{\mathrm{I}}\left(\xi, \eta, \zeta, t-\frac{r}{a}\right) \frac{\mathrm{I}}{r}\right\} d \sigma .
\end{aligned}
$$

Supponiamo che $\psi(x, y, z, t)$ rappresenti un potenziale di spostamento; allora (vedi $(I 7)) \psi_{2}(\xi, \eta, \zeta, t)$ rappresenterà il potenziale di velocità e $\psi_{I}(\xi, \eta, \zeta, t)$ (vedi (I 7$)$ ) rappresenterà la componente dello spostamento nel senso normale al contorno $\sigma$.

Supponiamo invece che $\psi(x, y, z, t)$ rappresenti un potenziale di velocità; in tale ipotesi $\psi_{2}(\xi, \eta, \zeta, t)$ sarà eguale ad $a^{2} \Theta$, essendo $\Theta$ il coefficiente di dilatazione e $\psi_{I}(\zeta, \eta, \zeta, t)$ rappresenterà la componente normale a $\sigma$ della velocità nei punti di $\sigma$. Potremo quindi enunciare la proposizione:

Il potenziale degli spostamenti [potenziale di velocità] in un istante $t$ nel punto $\mathrm{M} \equiv(x, y, z)$ dipende dal potenziale degli spostamenti [potenziale di velocità], dal potenziale di velocità [pressione, ovvero coefficiente di dilatazione] e dalla componente normale a $\sigma$ dello spostamento [velocità] in ciascun punto A di $\sigma$ in un istante $t^{\prime}$ anteriore all' istante $t$; e l'intervallo di tempo che decorre da t' a t ̀̀ uguale ad $r / a$, ciò̀ è proporzionale alla distanza $r$ del punto A del contorno dal punto M.

Questa stessa proposizione, in una con la precedente, può enunciarsi con altre parole.

Sia $\varphi(r+a t) / r$ il potenziale degli spostamenti [o di velocità] corrispondente ad un centro semplice: diremo che l'onda partita nell'istante $t^{\prime}$ dal centro $r=\mathrm{o}$ è giunta nell'istante $t=t^{\prime}+\frac{r}{a}$ nel punto $r$, e tale onda è individuata dal valore $\varphi\left(-a t^{\prime}\right)$.

Analogamente, nel caso di un centro doppio in cui il potenziale degli spostamenti [o di velocità] è $\left(\frac{\varphi^{\prime}(r-a t)}{r}-\frac{\varphi(r-a t)}{r^{2}}\right) \frac{\partial r}{\partial s}$ (vedi il I $)$, diremo 
che l'onda partita nell'istante $t$ dal centro e che giunge nell'istante $t=t^{\prime}+\frac{r}{a}$ nel punto $r$, è individuata dai valori $\varphi\left(-a t^{\prime}\right), \varphi^{\prime}\left(-a t^{\prime}\right)$.

Ciò premesso il teorema precedente potrà enunciarsi: Gli spostamenti [le velocità] del punto $\mathrm{M} \equiv(x, y, z)$ in un istante t possono considerarsi come dovuti ad onde partite dai punti A come centri negli istanti $t^{\prime}=t-\frac{r}{a}$ e gli elementi che individuano queste onde sono il valore del potenziale degli spostamenti [potenziale di velocità], del potenziale di velocità [pressione ovvero coefficiente di dilatazione], dello spostamento normale $a \sigma$ [velocità normale a $\sigma$ ] nei punti A negli istanti $t^{\prime}=t-\frac{r}{a}$.

12. Supponiamo ora che $\sigma$ sia una sfera col centro nel punto $x, y, z$. Prendendo la formula di KIRCHHOFF sotto la forma (2I) dovremo porre

$$
\frac{\partial r}{\partial n}=-\mathrm{I} \quad, \quad \frac{\partial \frac{\mathrm{I}}{r}}{\partial n}=\frac{\mathrm{I}}{r^{2}}
$$

quindi

$$
\begin{gathered}
\psi(x, y, z, t)=\frac{1}{4 \pi a} \int_{\sigma} \frac{\psi_{2}\left(\xi, \eta, \zeta, t-\frac{r}{a}\right)}{r} d \sigma \\
+\frac{1}{4 \pi} \int_{\sigma}\left\{\frac{\psi\left(\xi, \eta, \zeta, t-\frac{r}{a}\right)}{r^{2}}-\frac{\psi_{1}\left(\xi, \eta, \zeta, t-\frac{r}{a}\right)}{r}\right\} d \sigma
\end{gathered}
$$

e supponendo il raggio $r$ della sfera $=a t$

$$
\psi(x, y, z, t)=\frac{1}{4 \pi a} \int_{\sigma} \frac{\psi_{2}(\xi, \eta, \zeta, o)}{r} d \sigma+\frac{1}{4 \pi} \int_{\sigma}\left\{\frac{\psi(\xi, \eta, \zeta, 0)}{r^{2}}-\frac{\psi_{x}(\xi, \eta, \zeta, 0)}{r}\right\} d \sigma .
$$

Si consideri

$$
\frac{\partial}{\partial t} \int_{\sigma} \frac{\psi(\xi, \eta, \zeta, 0)}{r} d \sigma=\frac{\partial}{\partial t} \int_{\alpha} \psi(\xi, \eta, \zeta, 0) r d \alpha=\frac{\partial}{\partial t} \int_{\alpha} \psi(\xi, \eta, \zeta, 0) a t d \alpha
$$

essendo $\alpha$ la sfera di raggio ı. Avremo

$$
\frac{\partial}{\partial t} \int_{\sigma} \frac{\psi(\xi, \eta, \zeta, 0)}{r} d \sigma=\int_{\alpha}\left\{\psi(\xi, \eta, \zeta, 0) a d \alpha+\left(\frac{\partial \psi}{\partial \xi} \frac{\partial \xi}{\partial t}+\frac{\partial \psi}{\partial \eta} \frac{\partial \eta}{\partial t}+\frac{\partial \psi}{\partial \zeta} \frac{\partial \zeta}{\partial t}\right) a t\right\} d \alpha .
$$

$\mathrm{Ma}$

$$
\begin{gathered}
\frac{\partial \psi}{\partial \xi} \frac{\partial \xi}{\partial t}+\frac{\partial \psi}{\partial \eta} \frac{\partial \eta}{\partial t}+\frac{\partial \psi}{\partial \zeta} \frac{\partial \zeta}{\partial t}=a\left(\frac{\partial \psi}{\partial \xi} \frac{\partial \xi}{\partial(a t)}+\frac{\partial \psi}{\partial \eta} \frac{\partial \eta}{\partial(a t)}+\frac{\partial \psi}{\partial \zeta} \frac{\partial \zeta}{\partial(a t)}\right) \\
=a\left(\frac{\partial \psi}{\partial \xi} \frac{\partial \xi}{\partial r}+\frac{\partial \psi}{\partial \eta} \frac{\partial \eta}{\partial r}+\frac{\partial \psi}{\partial \zeta} \frac{\partial \zeta}{\partial r}\right)=-a\left(\frac{\partial \psi}{\partial \xi} \frac{\partial \xi}{\partial n}+\frac{\partial \psi}{\partial \eta} \frac{\partial \eta}{\partial n}+\frac{\partial \psi}{\partial \zeta} \frac{\partial \zeta}{\partial n}\right)=-a \psi(\xi, \eta, \zeta, 0) ;
\end{gathered}
$$


quindi

$$
\begin{gathered}
\frac{\partial}{\partial t} \int_{\sigma} \frac{\psi(\xi, \eta, \zeta, 0)}{r} d \sigma=a \int_{\alpha}\left\{\psi(\xi, \eta, \zeta, 0)-\psi_{I}(\xi, \eta, \zeta, 0) a t\right\} d \alpha \\
=a \int_{\sigma}\left\{\frac{\psi(\xi, \eta, \zeta, 0)}{r^{2}}-\frac{\psi_{1}(\xi, \eta, \zeta, 0)}{r}\right\} d \sigma
\end{gathered}
$$

onde tenendo presente la (22)

$$
\psi(x, y, z, t)=\frac{1}{4 \pi a}\left\{\int_{\sigma} \frac{\psi_{2}(\xi, \eta, \zeta, 0)}{r} d \sigma+\frac{\partial}{\partial t} \int_{\sigma} \frac{\psi(\xi, \eta, \zeta, o)}{r} d \sigma\right\} .
$$

Siccome i punti $\xi, \eta, \zeta$ appartengono alla sfera di raggio at e di centro $x, y, z$, potremo scrivere

$\xi=x+a t \operatorname{sen} \omega \cos \varphi \quad, \quad \eta=y+a t \operatorname{sen} \omega \operatorname{sen} \varphi \quad, \quad \zeta=z+a t \cos \omega$,

$$
d \sigma=r^{2} \operatorname{sen} \omega d \omega d \varphi=a^{2} t^{2} \operatorname{sen} \omega d \omega d \varphi
$$

onde

$$
\psi(x, y, z, t)
$$

$=\frac{I}{4 \pi}\left\{\int_{0}^{\pi} d \omega \int_{0}^{2 \pi} d \varphi \cdot \psi_{2}(x+a t \operatorname{sen} \omega \cos \varphi, y+a t \operatorname{sen} \omega \operatorname{sen} \varphi, z+a t \cos \omega, 0) t \operatorname{sen} \omega\right.$ $\left.+\frac{\partial}{\partial t} \int_{0}^{\pi} d \omega \int_{0}^{2 \pi} d \varphi \cdot \psi(x+a t \operatorname{sen} \omega \cos \varphi, y+a t \operatorname{sen} \omega \operatorname{sen} \varphi, z+a t \cos \omega, 0) t \operatorname{sen} \omega\right\}$.

13. La formula ora ottenuta è dovuta al Poisson. Essa ci dà l'integrale generale della equazione differenziale (I), giacché ci esprime la $\psi(x, y, z, t)$ mediante $\psi(x, y, z, 0)$ e $\left(\frac{\partial \psi}{\partial t}\right)_{t=0}$. Verifichiamo direttamente il risultato che abbiamo ottenuto come caso particolare della formula di KIRCHHOFF.

Dimostriamo cioè direttamente il teorema:

Essendo $\Phi(x, y, z), \Phi_{1}(x, y, z)$ due funzioni arbitrarie finite e continue insieme alle derivate prime e seconde, la funzione

$$
\psi(x, y, z, t)
$$

$=\frac{1}{4 \pi} \int_{0}^{\pi} d \omega \int_{0}^{2 \pi} d \varphi \Phi_{1}(x+a t \operatorname{sen} \omega \cos \varphi, y+a t \operatorname{sen} \omega \operatorname{sen} \varphi, z+a t \cos \omega) t \operatorname{sen} \omega$ $+\frac{\partial}{\partial t} \frac{1}{4 \pi} \int_{0}^{\pi} d \omega \int_{0}^{2 \pi} d \varphi \Phi(x+a t \operatorname{sen} \omega \cos \varphi, y+a t \operatorname{sen} \omega \operatorname{sen} \varphi, z+a t \cos \omega) t \operatorname{sen} \omega$ gode delle proprietà seguenti:

I ${ }^{\circ}$ soddisfa l'equazione differenziale

$$
\frac{\partial^{2} \psi}{\partial t^{2}}=a^{2} \Delta^{2} \psi
$$


$2^{\circ}$ soddisfa le condizioni

$$
\begin{gathered}
\psi(x, y, z, t)_{t=0}=\Phi(x, y, z) \\
\left(\frac{\partial \psi}{\partial t}\right)_{t=0}=\Phi_{x}(x, y, z) .
\end{gathered}
$$

Cominciamo dal provare che

$$
\psi^{\prime}(x, y, z, t)
$$

$=\frac{\mathrm{I}}{4 \pi} \int_{0}^{\pi} d \omega \int_{0}^{2 \pi} d \varphi \Phi_{1}(x+a t \operatorname{sen} \omega \cos \varphi, y+a t \operatorname{sen} \omega \operatorname{sen} \varphi, z+a t \cos \omega) t \operatorname{sen} \omega$ scddisfa l'equazione (26).

Si ponga per semplicità

(28) $\xi=x+a t \operatorname{sen} \omega \cos \varphi, \eta=y+a t \operatorname{sen} \omega \operatorname{sen} \varphi, \zeta=z+a t \cos \omega, \quad a t=r$ e si chiami $\alpha$ la sfera si raggio I. Allora potrà scriversi

$$
\psi^{\prime}(x, y, z, t)=\frac{t}{4 \pi} \int_{\alpha} \Phi_{x}(\xi, \eta, \zeta) d \alpha
$$

e derivando rispetto a $t$

$$
\frac{\partial \psi^{\prime}(x, y, z, t)}{\partial t}=\frac{1}{4 \pi} \int_{\alpha} \Phi_{1}(\xi, \eta, \zeta) d \alpha+\frac{t}{4 \pi} \int_{\sigma} \frac{\partial}{\partial t} \Phi_{1}(\xi, \eta, \zeta) d \alpha .
$$

Ora

$t \int_{\alpha} \frac{\partial}{\partial t} \Phi_{1}(\xi, \eta, \zeta) d \alpha=a t \int_{\alpha} \frac{\partial}{\partial r} \Phi_{1}(\xi, \eta, \zeta) d \alpha=\frac{1}{r} \int_{\sigma} \frac{\partial}{\partial r} \Phi_{1}(\xi, \eta, \zeta) d \sigma=\frac{1}{r} \int_{\dot{S}} \Delta^{2} \Phi_{x} d \mathrm{~S}$

chiamando $\sigma$ la superficie sferica di raggio $r$ e $\mathrm{S}$ lo spazio in essa racchiuso. Quindi

$$
\frac{\partial \psi^{\prime}(x, y, z, t)}{\partial t}=\frac{1}{4 \pi} \int_{\alpha} \Phi_{x}(\xi, \eta, \zeta) d \alpha+\frac{1}{4 \pi r} \int_{S} \Delta^{2} \Phi_{x} d S
$$

derivando nuovamente rispetto a $t$ troveremo,

$$
\frac{\partial^{2} \psi^{\prime}(x, y, z, t)}{\partial t^{2}}=\frac{\mathrm{I}}{4 \pi} \int_{\alpha} \frac{\partial}{\partial t} \Phi_{x}(\xi, \eta, \zeta) d \alpha-\frac{a}{4 \pi r^{2}} \int_{\mathrm{S}} \Delta^{2} \Phi_{x} d \mathrm{~S}+\frac{\mathrm{I}}{4 \pi r} \frac{\partial}{\partial t} \int_{\mathrm{S}} \Delta^{2} \Phi_{x} d \mathrm{~S} .
$$

$\mathrm{Ma}$

$$
\frac{I}{4 \pi} \int_{\alpha} \frac{\partial}{\partial t} \Phi_{I}(\xi, \eta, \zeta) d \alpha=\frac{a}{4 \pi r^{2}} \int_{\sigma} \frac{\partial}{\partial r} \Phi_{I}(\xi, \eta, \zeta) d \sigma
$$

e perciò

$$
\begin{gathered}
\frac{\partial^{2} \psi^{\prime}(x, y, z, t)}{\partial t^{2}}=\frac{a}{4 \pi r^{2}}\left\{\int_{\sigma} \frac{\partial}{\partial r} \Phi_{1}(\xi, \eta, \zeta) d \sigma-\int_{\mathrm{S}} \Delta^{2} \Phi_{1} d \mathrm{~S}\right\} \\
+\frac{\mathrm{I}}{4 \pi r} \frac{\partial}{\partial t} \int_{\mathrm{S}} \Delta^{2} \Phi_{1} d \mathrm{~S}=\frac{\mathrm{I}}{4 \pi r} \frac{\partial}{\partial t} \int_{\mathrm{S}} \Delta^{2} \Phi_{\mathrm{I}} d \mathrm{~S} .
\end{gathered}
$$


Abbiamo ora, chiamando $p$ il raggio vettore,

$$
\int_{S} \Delta^{2} \Phi_{1} d S=\int_{0}^{r} \rho^{2} d \rho \int_{\alpha} \Delta^{2} \Phi_{x} d \alpha=\int_{0}^{a t} \rho^{2} d \rho \int_{\alpha} \Delta^{2} \Phi_{x} d \alpha
$$

quindi

$$
\frac{\partial}{\partial t} \int_{\mathrm{S}} \Delta^{2} \Phi_{1} d \mathrm{~S}=a r^{2} \int_{\alpha} \Delta^{2} \Phi_{1} d \alpha
$$

d'onde

$$
\frac{\partial^{2} \cdot \psi^{\prime}(x, y, z, t)}{\partial t^{2}}=\frac{a r}{4 \pi} \int_{\alpha} \Delta^{2} \Phi_{1} d \alpha=\frac{a^{2} t}{4 \pi} \int_{\alpha} \Delta^{2} \Phi_{1} d \alpha .
$$

I1 $\Delta^{2}$ che comparisce nelle formule precedenti è

$$
\frac{\partial^{2}}{\partial \xi^{2}}+\frac{\hat{\partial}^{2}}{\partial \eta^{2}}+\frac{\hat{\partial}^{2}}{\partial \zeta^{2}} \text {; }
$$

a cagione delle (28) avremo evidentemente

$$
\frac{\partial^{2}}{\partial \xi^{2}}+\frac{\partial^{2}}{\partial \eta^{2}}+\frac{\partial^{2}}{\partial \zeta^{2}}=\frac{\partial^{2}}{\partial x^{2}}+\frac{\hat{o}^{2}}{\partial y^{2}}+\frac{\partial^{2}}{\partial z^{2}}
$$

onde l'operazione $\Delta^{2}$ potrà essere permutata con l'operazione $\int_{\alpha}$ e quindi

$$
\frac{\partial^{2} \psi^{\prime}(x, x, z, t)}{\partial t^{2}}=a^{2} \Delta^{2}\left\{\frac{t}{4 \pi} \int_{\alpha} \Phi_{1}(\xi, \eta, \zeta) d \alpha\right\}=a^{2} \Delta^{2} \psi^{\prime} \text {. C. D. D. }
$$

Facciamo nelle (29), (30), (3I), $t=0$, si troverà

$$
\begin{cases}\left(32^{\prime}\right) & \left.\psi^{\prime}(x, y, z, t)\right)_{t=0}=0 \\ \left(32^{\prime \prime}\right) & {\left[\frac{\partial \psi^{\prime}(x, y, z, t)}{\partial t}\right]_{t=0}=\frac{1}{4 \pi} \Phi_{1}(x, y, z) \int_{\sigma} d \alpha=\Phi_{1}(x, y, z)} \\ \left(32^{\prime \prime \prime}\right) & {\left[\frac{\hat{o}^{2} \psi^{\prime}(x, y, z, t)}{\partial t^{2}}\right]_{t=0}=0 .}\end{cases}
$$

Si ponga ora

$$
\psi^{\prime \prime}(x, y, z, t)
$$

$=\frac{1}{4 \pi} \int_{0}^{\pi} d \omega \int_{0}^{2 \pi} d \varphi \Phi(x+a t \operatorname{sen} \omega \cos \varphi, y+a t \operatorname{sen} \omega \operatorname{sen} \varphi, z+a t \cos \omega) t \operatorname{sen} \omega$.

Questa funzione sarà perfettamente analoga alla (27), solo invece di dipendere dalla funzione arbitraria $\Phi_{\mathrm{r}}$ dipenderà da $\Phi$. Essa godrà quindi di tutte le proprietà analoghe a quelle della (27) e perciò essa pure soddisfarà l'equazione (26) e (vedi (32))

$$
\left\{\begin{array}{l}
\psi^{\prime \prime}(x, y, z, t) t_{t=0}=0 \\
{\left[\frac{\partial \psi^{\prime \prime}(x, y, z, t)}{\partial t}\right]_{t=0}=\Phi(x, y, z)} \\
{\left[\frac{\partial^{2} \psi^{\prime \prime}(x, y, z, t)}{\partial t^{2}}\right]_{t=0}=0 .}
\end{array}\right.
$$


Ma noi abbiamo (vedi (25))

$$
\psi(x, y, z, t)=\psi^{\prime}(x, y, z, t)+\frac{\partial}{\partial t} \psi^{\prime \prime}(x, y, z, t)
$$

dunque anche $\psi$ verificherà la $(26)$ e a cagione delle $(32)$ e (33) sarà

$$
\begin{aligned}
& \psi(x, y, z, t)_{t=0}=\Phi(x, y, z) \\
& {\left[\frac{\partial \psi(x, y, z, t)}{\partial t}\right]_{t=0}=\Phi_{1}(x, y, z)}
\end{aligned}
$$

Relativamente al significato della formula di PoIsson basta riferirsi a quello che si disse riguardo alle formule di KIRCHHOFF (vedi il $§$ I I). Quindi ogni qual volta si valuta il potenziale degli spostamenti e il potenziale di velocità mediante la formula di POISSON, ciò significa che si riguarda il moto in un punto A in un dato istante t come dovuto alla sovrapposizione di un doppio sistema di onde partite nel tempo iniziale dai punti di una superficie sferica di raggio at, col centro in $\mathrm{A}$, le quali sono individuate dagli elementi relativi allo stato di moto dei punti della superficie sferica nell'origine dei tempi $\left(^{*}\right)$.

$\left(^{*}\right)$ Nell'originale di questa Nota è qui preannunciata una "continuazione" che non figura in alcuno dei successivi volumi del "Nuovo Cimento". [N. d. R.]. 